\title{
Failure of Nielsen's theorem in higher dimensions
}

\author{
By \\ Frank Raymond and Leonard L. Scott *)
}

The following is known for closed orientable surfaces. If $H: M \rightarrow M$ is a map whose $n$-th power is homotopic to the identity, then $H$ is homotopic to a homeomorphism $K$ with $K^{n}=$ identity. The result is known as Nielsen's Theorem on finite mapping classes. There are doubts (see [8]) as to the correctness of all parts of Nielsen's arguments in [6]. Different (using complex analysis and the Smith theorems) and valid proofs have been given independently by Fenchel and Macbeath. Because we are dealing with surfaces, each self homotopy equivalence is homotopic to a diffeomorphism, and homotopic diffeomorphisms are diffeotopic. Consequently, Nielsen's theorem may be equivalently stated as follows: If $H: M \rightarrow M$ is a diffeomorphism whose $n$-th power is homotopic to the identity then $H$ is diffeotopic to a diffeomorphism $K$ with $K^{n}=$ identity. The theorem is extremely useful in studying periodic maps on 3-manifolds, and there are obvious applications in surface theory.

The question has been raised as to what extent Nielsen's theorem holds for aspherical manifolds of dimensions greater than 2. An aspherical manifold is a closed manifold whose universal covering is contractible. Aspherical manifolds are therefore closed manifolds which are also $K(\pi, 1)$ 's. An important and interesting class of aspherical manifolds (generalizing the tori) are the nil-manifolds. A nil-manifold is simply the quotient of a connected contractible nilpotent Lie group by a uniform discrete subgroup.

We shall show that in dimensions greater than 2 there exist closed nil-manifolds for which Nielsen's theorem fails in a very strong sense. Specifically

Theorem 1. For each $m>2$ there exist closed m-dimensional nil-manifolds, $M^{m}$, and diffeomorphisms $H: M \rightarrow M$ so that $H^{2}$ is diffeotopic to the identity but $H$ is not homotopic to any homeomorphism $K$ so that $K^{2}=$ identity.

The method of proof is not restricted to period 2. In fact in Theorem 2 we shall describe examples for any period $k$. In all of these examples the nil-manifolds are fiber bundles fibered over the circle with fiber an $(m-1)$-torus.

We would now like to put our negative results into perspective with some known positive results.

*) Research partially supported by the National Science Foundation. 
Let $H(X)$ denote the (topological) group of self-homeomorphisms of a closed manifold $X$ with the compact-open topology. Let $E(X)$ denote the $H$-space of selfhomotopy equivalences of $X$ and $\psi: E(X) \rightarrow$ Out $\pi_{1}(X, x)$ be the map which assigns to each self-homotopy equivalence the outer automorphism induced on the fundamental group. If $E_{0}(X)$ denotes the path-component of the identity of $E(X)$, then $E(X) / E_{0}(X)$ is a discrete group. (This discreteness may be deduced, for example, from [5; Theorem 4.1].) Since $\left.\psi\right|_{E_{0}(X)}$ is trivial, $\psi$ induces a homomorphism

$$
\psi^{\prime}: E(X) / E_{0}(X) \rightarrow \operatorname{Out}\left(\pi_{1}(X, x)\right) \text {. }
$$

Let $j: H(X) \stackrel{c}{\rightarrow} E(X)$ denote the natural inclusion. The composition

$$
\psi \circ j: H(X) \rightarrow \text { Out } \pi_{1}(X, x)
$$

is a homomorphism.

If $X$ is a $K\left(\pi_{1}(X), 1\right)$ then $\psi^{\prime}$ is bijective. If $X$ is a nil-manifold it is known that $\psi \circ j$ is surjective. That is, every homotopy equivalence of a closed nil-manifold is homotopic to a homeomorphism (in fact a diffeomorphism).

In [2] it was shown that for "most" bundles $B$, which fiber over the circle with a $k$-torus $T^{k}$ as fiber, the homomorphism

$$
\psi: H(B) \rightarrow \operatorname{Out}\left(\pi_{1}(B, b)\right)
$$

is onto and splits. That is, there exists a homomorphism $\chi: \operatorname{Out}\left(\pi_{1}(B, b)\right) \rightarrow H(B)$ so that $\psi \circ \chi=$ identity. Our examples will be bundles fibered over the circle with fiber a torus. Therefore the "most" can not be improved to all. The theorem of [2] has been extended to what is called injective Seifert fiber spaces, $\left(T^{k}, X, \varphi\right)$. It is not needed that $X$ is even a manifold. A certain subgroup $L$ of Out $\pi_{1}(X, x)$ is characterized and it is shown that the homomorphism

$$
\psi: H(X) \rightarrow \operatorname{Out} \pi_{1}(X, x)
$$

has image containing $L$. In fact for any finite subgroup $F$ of $L$ we may actually lift $F$ back into $H(X)$. See $[3 ; \S 9$, Th. 12 , Cor. 1$]$.

Since our examples are injective Seifert fiberings our homeomorphisms $H$ will have image in Out $\pi_{1}(X, x)$ outside of the subgroup $L$. In fact, the obstruction to a Nielsen theorem in each of our examples is forced algebraically by the obstruction to a certain group extension. If one considers fiberings over the circle where the fundamental group of the fiber has trivial center, this algebraic difficulty is avoided. Recently, J. Tollefeson has found the following generalization of Nielsen's theorem in dimension 3, [7]: Let $p$ be a prime and $M^{3}$ a closed 3-manifold with $H_{1}\left(M^{3} ; Q\right) \cong Q$ and which fibers over the circle with fiber a closed surface having negative Euler characteristic. Then, if $h: M \rightarrow M$ is a map so that $h^{p}$ is homotopic to the identity, there exists a $P L$ homeomorphism $K: M \rightarrow M$ so that $K^{p}=$ identity.

In the remarks following Theorem 1 we shall examine further the differences between our examples and Tollefson's positive results. Now let us turn to the

Proof of Theorem 1. We shall describe the case $m=3$ and then indicate how the description can be extended to all dimensions greater than 3 . 
Let $\pi$ be a group presented as follows

$$
\pi=\left\langle v_{1}, v_{2}, n \mid\left[v_{1}, v_{2}\right]=1, n v_{1} n^{-1}=v_{1} v_{2}^{2}, n v_{2} n^{-1}=v_{2}\right\rangle .
$$

We may think of $\pi$ as the semi-direct product $\left(Z_{1} \times Z_{2}\right) \circ \mathbb{Z}$. The $v_{i}$ are the generators of the infinite cyclic groups $Z_{i}$, and $n$ is the generator of $\mathbb{Z}$. The defining action of the generator $n$ on $Z_{1} \times Z_{2}$ is given by the matrix $\left(\begin{array}{ll}1 & 0 \\ 2 & 1\end{array}\right)$.

On $\pi$ we wish to describe an automorphism $t$ so that $t^{2}=$ conjugation by $n$. Put

$$
t\left(v_{1}\right)=v_{1}^{-1} v_{2}^{-1}, \quad t\left(v_{2}\right)=v_{2}^{-1}, \quad t(n)=v_{2} n .
$$

This defines an automorphism $t: \pi \rightarrow \pi$ since the relations are preserved. On $Z_{1} \times Z_{2}$ it restricts to $\left(\begin{array}{rr}-1 & 0 \\ -1 & -1\end{array}\right)$. Now $t^{2}$ is the automorphism of $\pi$ given by conjugation by $n$ since

$$
t^{2}\left(v_{1}\right)=t\left(v_{1}^{-1} v_{2}^{-1}\right)=v_{1} v_{2}^{2}, t_{2}\left(v_{2}\right)=v_{2}, t^{2}(n)=n .
$$

Moreover, $t$ itself is not inner because $t$ acts non-trivially on $v_{2}$ which generates the center of $\pi$. Therefore $t$ generates an element $t^{\prime}$ of order 2 in Out $\pi$.

Define an injection

$$
\psi: \mathbb{Z} / 2 \mathbb{Z} \rightarrow \text { Out } \pi
$$

by sending the generator of $\mathbb{Z} / 2 \mathbb{Z}$ onto $t^{\prime}$. This defines an abstract kernel $(\mathbb{Z} / 2 \mathbb{Z}, \pi, \psi)$.

We claim that

Proposition. The abstract kernel $(\mathbb{Z} / 2 \mathbb{Z}, \pi, \psi)$ has no extension.

Proof. Suppose such an extension $E$ exists, then we have the commutative diagram:

$$
\begin{aligned}
& 1 \rightarrow \operatorname{Inn} \pi \rightarrow \text { Aut } \pi \rightarrow \text { Out } \pi \rightarrow 1 \\
& 1 \rightarrow \begin{array}{l}
\uparrow \\
1 \rightarrow \stackrel{\uparrow}{\pi}
\end{array} \quad \rightarrow \stackrel{\uparrow}{\uparrow} / 2 \mathbb{Z} \rightarrow 1 .
\end{aligned}
$$

There exists an element $e \in E$ whose image is exactly $t$. Let $s=e^{2}$. Then $s \in \pi$ and $t(s)=e e^{2} e^{-1}=e^{2}=s$. Now the image of $s$ in Aut $\pi$ is conjugation by $s$ and it must also equal conjugation by $n$. Thus $s=c n$, for some central element $c$ of $\pi$. Since $t(n) \neq n$, this element $c$ is non-trivial.

The general form of the action of $t$ is

$$
t\left(v_{1}^{b} v_{2}^{c} n^{a}\right)=\left(v_{1}^{-1} v_{2}^{-1}\right)^{b} v_{2}^{-c}\left(n v_{2}\right)^{a}=v_{1}^{-b} v_{2}^{a-b-c} n^{a} .
$$

For a fixed point of $t$ we have

$$
b=-b, \quad c=a-b-c \text { or } b=0, \quad 2 c=a .
$$

Thus the fixed point group of $t$ is $\left\langle v_{2} n^{2}\right\rangle$. Hence $s \in\left\langle v_{2} n^{2}\right\rangle$ could not possibly be equal to $c n$, and so no extension exists corresponding to the abstract kernel defined by $t^{\prime}$. This completes the proof of the proposition.

Let us now describe $M^{3}$ so that $\pi_{1}\left(M^{3}, *\right)=\pi$. $M^{3}$ will be defined as a bundle over the circle with fiber a 2-torus $T^{2}$. We may describe it as the quotient of $T^{2} \times \mathbb{R}^{1}$ 
by the diagonal action of $\mathbb{Z}$ given (on a generator) by

$$
\left(z_{1}, z_{2}, r\right) \rightarrow\left(z_{1}, z_{1}^{2} z_{2}, r-1\right),
$$

where $z_{i} \in S^{1}, r \in \mathbb{R}$. Denote elements of the orbit space $M^{3}=T^{2} \times_{\mathbb{Z}} \mathbb{R}^{1}$ by $\left(\left(z_{1}, z_{2}, r\right)\right)$.

Define $H: M^{3} \rightarrow M^{3}$ by

$$
\left(\left(z_{1}, z_{2}, r\right)\right) \rightarrow\left(\left(z_{1}^{-1}, z_{1}^{-1} z_{2}^{-1} e^{2 \pi i r}, r\right)\right) \text {. }
$$

This is well-defined since it can be defined on $T^{2} \times \mathbb{R}^{1}$ so that it commutes with the action of $\mathbb{Z}$ :

$$
\begin{aligned}
\left(z_{1}, z_{2}, r\right) \longrightarrow & \left(z_{1}, z_{1}^{2}, z_{2}, r-1\right) \\
\downarrow & \left(z_{1}^{-1}, z_{1}^{-1} z_{1}^{-2} z_{2}^{-1} e^{2 \pi i r(r-1)}, r-1\right) \\
& \\
\left(z_{1}^{-1}, z_{1}^{-1} z_{2}^{-1} e^{2 \pi i r}, r\right) \longrightarrow & \left(z_{1}^{-1}, z_{1}^{-2} z_{1}^{-1} z_{2}^{-1} e^{2 \pi i r}, r-1\right) .
\end{aligned}
$$

Now, $H^{2}$ is given by

$$
\left(\left(z_{1}, z_{2}, r\right)\right) \rightarrow\left(\left(z_{1}, z_{1}^{2} z_{2}, r\right)\right) .
$$

We observe that the point $a=((1,1,0)) \in M^{3}$ is left fixed by $H$, and $H_{*}$ induces the automorphism $t$ on $\pi_{1}(M, a)$. Furthermore, it is clear that $H^{2}$ defines, on $M^{3}$, a diffeomorphism which induces on $\pi_{1}(M, a)$ the inner automorphism given by conjugation by $n$. In fact $H^{2}$ is diffeotopic to the identity. We may define the isotopy by

$$
\mathcal{O}_{\alpha}:\left(\left(z_{1}, z_{2}, r\right)\right) \rightarrow\left(\left(z_{1}, z_{1}^{2} z_{2}, r-\alpha\right)\right), 0 \leqq \alpha \leqq 1 .
$$

This $\operatorname{map} \mathcal{O}_{\alpha}$ is well defined and yields the desired isotopy.

Now if there exists a homeomorphism $K: M^{3} \rightarrow M^{3}$ homotopic to $H$ with $K^{2}=$ identity, then there exists an extension of the form:

$$
1 \rightarrow \pi_{1}(M, a) \rightarrow E \rightarrow \mathbb{Z} / 2 \mathbb{Z} \rightarrow 1 \text {. }
$$

One may think of $E$ as acting on the universal covering space $\tilde{M}^{3} \approx \mathbb{R}^{3}$ of $M^{3}$. In fact, an explicit construction of the action $\left(E, \tilde{M}^{3}\right)$ is given in $[1 ; \S 2]$. Furthermore, the abstract kernel associated to this extension is precisely our $(\mathbb{Z} / 2 \mathbb{Z}, \pi, \psi)$ which we know can not exist. Thus, $K^{2}$ can not be the identity.

Finally, that $M^{3}$ is a nil-manifold (the quotient of an analytic nilpotent lie group by a uniform discrete subgroup) is well known. In fact the Lie group here is just the group of real upper triangular matrices of the form $\left(\begin{array}{lll}1 & * & * \\ 0 & 1 & * \\ 0 & 0 & 1\end{array}\right)$. This completes the
proof of Theorem 1 for $m=3$.

Let us now describe how the result can be extended to all dimensions greater then 3 . We replace $M^{3}$ by $M^{3} \times T^{m-3}$. We define $t$ on $\pi \times \mathbb{Z}^{m-3}$ by letting it act trivially on $\mathbb{Z}^{m-3}$ and as before on $\pi$. We may carry out the remaining part of the argument completely analogously to the case $m=3$. This completes the proof of Theorem 1 .

Remarks. 1. Generalizing Theorem 1 to even periods. We would like to thank the referee and Darryl MeCullough who kindly pointed out that slight modifications of 
our construction in Theorem 1 also yield 3-dimensional nil-manifolds for which Nielsen's theorem fails for any even period. We shall describe their generalization now.

Let $j$ and $t$ be arbitrary positive integers. In strict analogy to the construction of Theorem 1 put

$$
\pi=\left\langle v_{1}, v_{2}, n \mid\left[v_{1}, v_{2}\right]=1, n v_{1} n^{-1}=v_{1} v_{2}^{2 k j}, n v_{2} n^{-1}=v_{2}\right\rangle .
$$

Let $t: \pi \rightarrow \pi$ be defined by

$$
t\left(v_{1}\right)=v_{1}^{-1} v_{2}^{-j}, \quad t\left(v_{2}\right)=v_{2}^{-1}, \quad t(n)=v_{2} n .
$$

It follows that

$$
\begin{array}{lll}
t^{2 q}\left(v_{1}\right)=v_{1} v_{2}^{2 q j}, & t^{2 q}\left(v_{2}\right)=v_{2}, & t^{2 q}(n)=n, \\
t^{2 q+1}\left(v_{1}\right)=v_{1}^{-1} v_{2}^{-(2 q+1) j}, & t^{2 q+1}\left(v_{2}\right)=v_{2}^{-1}, & t^{2 q+1}(n)=v_{2} n,
\end{array}
$$

and $t^{2 k}$ is the inner automorphism $x \rightarrow n x n^{-1}$. It is easily checked that $t^{r}$ is not an inner automorphism for all $r, 0<r<2 k$.

Let $\psi: \mathbb{Z} / 2 k \mathbb{Z} \rightarrow$ Out $\pi$ be the monomorphism defined by assigning to $t$ its outer automorphism class.

Proposition. The abstract kernel $(\mathbb{Z} / 2 \mathbb{Z}, \pi, \psi)$ has no extension.

The proof is the same as in the earlier proposition.

We construct $M^{3}(k, j)$ as a bundle over the circle with fiber a torus with monodromy $\left(\begin{array}{cc}1 \\ 2 k j & 1\end{array}\right)$. The formula for $H$ is $\left(\left(z_{1}, z_{2}, r\right)\right) \rightarrow\left(\left(z_{1}^{-1}, z_{1}^{-j} z_{2}^{-1} e^{2 \pi i r}, r\right)\right)$. Once again $((1,1,0))=a$ is left fixed and $H_{*}$ induces the automorphism $t$ on $\pi_{1}(M, a) . H^{2 k}$ is diffeotopic to the identity via

$$
\mathcal{O}_{\alpha}:\left(\left(z_{1}, z_{2}, r\right)\right) \rightarrow\left(\left(z_{1}, z_{1}^{2 k j} z_{2}, r-\alpha\right)\right),
$$

and $H$ is not homotopic to any homeomorphism $K$ so that $K^{2 k}=$ identity. We obtain examples in higher dimensions by multiplying by a torus as before.

Using our methods, J.Tollefson has suggested other 3-dimensional aspherical manifolds for which Nielsen's theorem fails. P. E. Conner informs us that he was also aware of important aspects of our examples.

2. $M^{3}(k, j)$ as a Seifert manifold. For $k=j=1, M^{3}(k, j)$ is the manifold of Theorem 1. It may be useful for the reader to observe that $M^{3}(k, j)$ can also be viewed alternatively as a classical 3-dimensional Seifert fiber space. In particular, it is a principal circle bundle over the torus $T^{2}$ with first Chern class - $(2 k j)$ times the fundamental cocycle in $H^{2}\left(T^{2} ; \mathbb{Z}\right)$. The "canonical" presentation of $\pi_{1}\left(M^{3}\right)$ as a Seifert fiber space is

$$
\left\langle v_{1}, v_{2}, n \mid\left[v_{1}, v_{2}\right]=1,\left[n, v_{2}\right]=1, n v_{1} n^{-1} v_{1}^{-1}=v_{2}^{2 k j}\right\rangle \text {. }
$$

Here the generator $v_{2}$ represents the generator of the fundamental group of the circle fiber.

3. Out $\pi_{1} M^{3}(k, j)$. We may identify our automorphism $t$ and its outer outermorphism class among the full group of automorphisms and outer automorphisms of $\pi_{1} M^{3}(k, j)$. In $[1, \S 8]$ it is shown that

$$
\begin{aligned}
& \operatorname{Aut}\left(\pi_{1}\left(M^{3}(k, j)\right)\right)=(\mathbb{Z} \oplus \mathbb{Z}) \circ G L(2, \mathbb{Z}), \\
& \operatorname{Out}\left(\pi_{1}\left(M^{3}(k, j)\right)\right)=((\mathbb{Z} / 2 k j \mathbb{Z}) \oplus(\mathbb{Z} / 2 k j \mathbb{Z})) \circ G L(2, \mathbb{Z}) .
\end{aligned}
$$


Every homotopy equivalence of $M^{3}(k, j)$ can be deformed to a (Seifert) fiber preserving diffeomorphism (see [2]). The automorphism $t$ actually has projections onto both parts of the semi-direct product in Out $\pi$. However, it is more relevant for our examples to regard $M^{3}(k, j)$ as a fiber bundle over the circle. Every outer automorphism of $\pi$ which arises from an automorphism which is invariant on the group generated by $v_{1}$ and $v_{2}$ and which induces the identity on the quotient group (for example our $t$ ) can be realized by a diffeomorphism which moves only along the toral fibers, such as our $H$, (see $[3 ; \S 7]$ ). The subgroup of such outer automorphisms is isomorphic to

$$
(\mathbb{Z} \oplus \mathbb{Z} / 2 k j \mathbb{Z}) \circ((\mathbb{Z} / 2 k j \mathbb{Z}) \times(\mathbb{Z} / 2 \mathbb{Z}))
$$

which follows from $[1 ; 4.5]$. In terms of an obvious coordinate representation $\psi(t)=$ $=(0,1) \circ(j \times e)$, where $j \times e=\left(\begin{array}{cc}-1 & 0 \\ -j & -1\end{array}\right)$ and the action is the usual action. What we believe is significant here is that $\psi(t)$ has coordinate projections on each factor of the semi-direct product. If $\psi(t)$ were an element of either factor, then the abstract kernel would determine an extension and we would also be able to find diffeomorphisms $K$ so that $K^{2 k}=$ identity.

(In Tollefson's positive results ([7]) the outer automorphism group of $\pi_{1}\left(M^{3}\right)$ is really, in some sense, simpler than encountered in our examples. For it arises solely from the normalizer of the mapping class of the monodromy homeomorphism in the outer automorphism group of the fundamental group of the fiber, see $[1 ; \S 4]$. Consequently, two homotopy equivalences of $M^{3}$ which induce the same outer automorphism on a fiber are homotopic. One may, as Tollefson successfully does, try to extend a periodic map on a surface to $M^{3}$. In our examples, the diffeomorphisms $H$ and $H^{\prime}:\left\langle z_{1}, z_{2}, r\right\rangle \rightarrow\left\langle z_{1}^{-1}, z_{1}^{-j} z_{2}^{-1}, r\right\rangle$ agree on the fiber over $\langle 1,1,0\rangle$, but $H$ and $H^{\prime}$ are not homotopic in $M$.)

4. Poincaré Complexes. We may also ask if it is impossible to find a periodic homeomorphism $K$ on some Poincaré complex $X$ having the homotopy type of $M^{m}$. Actually, we have shown even more: If $X$ is a space so that $\pi_{1}\left(X, x_{0}\right) \cong \pi_{1}\left(M^{m}, a\right)$ and $t$ is the automorphism used in the theorem then there exists no homeomorphism $K$ with $K^{2 k}=$ identity and $K_{*}$ representing the same outer automorphism represented by $t$. For, if there were such a homeophormism $K$, then we would be able to construct the extension from the abstract kernel $(\mathbb{Z} / 2 k j \mathbb{Z}, \pi, \psi)$ by $[1 ; \S 2]$.

Periods greater than 2. The generalization from $M^{3}$ to $M^{3}(k, j)$ does not appear to work for odd periods. To obtain failure for all periods another modification of the method of Theorem 1 will be used in the proof of Theorem 2 .

Theorem 2. For each $k>1$, there exist closed $(2 k-1)$ dimensional nil-manifolds $M$ and diffeomorphisms $H: M \rightarrow M$ so that $H^{k}$ is diffeotopic to the identity (and $H^{l}$ is not homotopic to the identity for $0<l<k$ ) but $H$ is not homotopic to any homeomorphism $K$ with $K^{k}=$ identity.

Actually, the dimensions of $M$ are larger than necessary. We may modify the construction to bring the dimensions down to at least $3+(k-1)$. As in Theorem 1 , once one produces an example, one gets examples for that $k$ in all higher dimensions. 
Sketch of Proof. The construction and argument is a generalization of that used in Theorem 1. We take

$$
\begin{aligned}
& V_{1}=\mathbb{Z}^{k-1}=k-1 \text { copies of } \mathbb{Z}, \\
& V_{2}=\mathbb{Z}^{k-1}, \\
& V=V_{1} \times V_{2} .
\end{aligned}
$$

We form $\pi$ as the semi-direct product of $V$ with $\mathbb{Z}=\langle n\rangle$, where $n$ acts on $V$ by

$$
\left(\begin{array}{rr}
I & 0 \\
k I & I
\end{array}\right)
$$

We define $t$ on $V$ by

$$
\left(\begin{array}{ll}
C & 0 \\
C & C
\end{array}\right)
$$

where $C$ is the companion matrix of $\frac{x^{k}-1}{x-1}$. That is,

$$
C=\left(\begin{array}{rrrrr}
0 & 1 & 0 \ldots & & 0 \\
0 & 0 & 1 \ldots & & . \\
. & & & & . \\
. & & & & . \\
. & & 0 & 1 & 0 \\
0 & 0 & \ldots & 0 & 0 \\
-1 & -1 & \ldots & -1 & -1
\end{array}\right)
$$

Its characteristic polynomial is $\frac{x^{k}-1}{x-1}$. We put $t(n)=w n$ where $w \in V_{2}$ generates $V_{2}$ as a $\mathbb{Z}[C]$ module.

We claim $t$ is an automorphism of $\pi$ because the action of $t$ on $V$ commutes with that of $n$, and the element $w n=t(n)$ acts as $n$ on $V$ and also generates a com-

plementary copy of $\mathbb{Z}$ to $V$ in $\pi$.
Now $t^{k}$ on $V$ is conjugation by $n$, since $\left(\begin{array}{ll}C & 0 \\ C & C\end{array}\right)^{k}=\left(\begin{array}{rr}I & 0 \\ k I & I\end{array}\right)$. Also,

$$
t^{k}(n)=t^{k-1}(w) \cdots t(w) w n=\left(C^{k-1}+\cdots+1\right)(w) \cdot n=n .
$$

Therefore $t^{t}$ is conjugation by $n$ on all of $\pi$.

Once again we want to show that the $8(\pi) n$ contains no element of $\pi$ fixed by $t$, where $8(\pi)$ denotes the center of $\pi$. Let $v_{1} \in V_{1}, v_{2} \in V_{2}$, then

$$
t\left(v_{1} v_{2} n^{a}\right)=v_{1} v_{2} n^{a}
$$

if and only if

$$
t\left(v_{1}\right) C\left(v_{2}\right) w^{a}=v_{1} v_{2} .
$$

Then, $t\left(v_{1}\right) \equiv v_{1}\left(\bmod V_{2}\right)$ which implies $C\left(v_{1}\right) \equiv v_{1}\left(\bmod V_{2}\right)$ and so $C\left(v_{1}\right)=v_{1}$. This forces $v_{1}=0$ since 1 is not an eigenvalue of $C$. Now we have

$$
w^{a}=v_{2} C^{-1}\left(v_{2}\right)=(1-C)\left(v_{2}\right) \text {. }
$$


Put $v_{2}=\left(\sum_{i=0}^{k-1} z_{i} C^{i}\right)(w)$ where the $z_{i}$ 's are integers. Such an expression for an element of $V_{2}$ is unique up to the addition of an expression $\left(m \sum_{i=0}^{k-1} C^{i}\right)(w), m$ an integer (consider the homomorphism from the group algebra for the cyclic group of order $k$ onto $\mathbb{Z}[C])$. We have

$$
\left(a C^{0}\right)(w)=w^{a}=(1-C)\left(v_{2}\right)=\sum_{i=0}^{k-1}\left(z_{i}-z_{i-1}\right) C^{i}(w)
$$

where $z_{-1}=z_{k-1}$. Thus there is an integer $m$ with $a=z_{0}-z_{-1}+m$ and

$$
0=z_{1}-z_{0}+m=\cdots=z_{k-1}-z_{k-2}+m \text {. }
$$

Adding all these expressions together we get $a=k m$. Thus $a$ is divisible by $k$, and in particular $a \neq 1$ so $3(\pi) n$ contains no element of $\pi$ fixed by $t$. Consequently, we have shown that the abstract kernel $(\mathbb{Z} / k \mathbb{Z}, V \circ \mathbb{Z}, \psi)$ has no extension, where $\psi:(\mathbb{Z} / k \mathbb{Z}) \rightarrow$ Out $(V \circ \mathbb{Z})$ is induced by the automorphism $t$.

The geometric construction proceeds as in the proof of Theorem 1. Corresponding to the group $\pi=V \circ \mathbb{Z}$, we take $M^{2 k-1}=\left(T^{k-1} \times T^{k-1}\right) \times_{\mathbb{Z}} \mathbb{R}^{1}$, where this is the quotient of $T^{2 k-2} \times \mathbb{R}^{1}$ by the diagonal action of $\mathbb{Z}$ given on a generator by

$$
\left(z_{1}, \ldots, z_{k-1}, y_{1}, \ldots, y_{k-1}, r\right) \rightarrow\left(z_{1}, \ldots, z_{k-1}, z_{1} k_{y_{1}}, \ldots, z_{k-1} k_{y_{k-1}}, r-1\right)
$$

$z_{i}, y_{j} \in S^{1}, r \in \mathbb{R}$. We express $w$ in terms of our standard basis for $\nabla_{2}$ by

$$
w=w_{1} v_{2,1}+\cdots+w_{k-1} v_{2, k-1} \text {. }
$$

We now define $H$ on $M^{2 k-1}$ so that the induced automorphism on the fundamental group is given by $t$ by using the $2 k-1$ matrix:

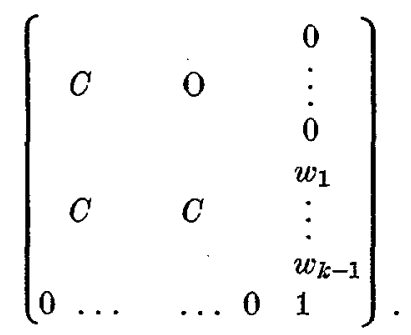
[For example, if $k=3$, then $C=\left(\begin{array}{rr}0 & 1 \\ -1 & -1\end{array}\right)$. For this case we may choose
$w=v_{2,1} \in V_{2}$. Then we define

$$
\left.H\left(\left(z_{1}, z_{2}, y_{1}, y_{2}, r\right)\right)=\left(\left(z_{2}, z_{1}^{-1} z_{2}^{-1}, z_{2} y_{2} e^{2 \pi i r},\left(z_{1} z_{2} y_{1} y_{2}\right)^{-1}, r\right)\right) \cdot\right]
$$

The diffeomorphism $H^{k}$ may be isotoped to the identity in a manner similar to that employed in the proof of Theorem 1 because

$$
\begin{aligned}
& H^{k}\left(\left(z_{1}, \ldots, z_{k-1}, y_{1}, \ldots, y_{k-1}, r\right)\right)= \\
& \quad=\left(\left(z_{1}, \ldots, z_{k-1}, z_{1}{ }^{k} y_{1}, \ldots, z_{k-1}{ }^{k} y_{k-1}, r\right)\right) .
\end{aligned}
$$

Of course, $H$ is not homotopic to any homeomorphism $K$ so that $K^{k}=$ identity, for 
otherwise we would have to have an extension for our abstract kernel $(\mathbb{Z} \mid k \mathbb{Z}, V \circ \mathbb{Z}, \psi)$ as in the proof of Theorem 1. This completes the proof of Theorem 2.

We have seen that the lack of a Nielsen theorem in our examples is forced algebraically by the lack of an extension. It would be very interesting to find an example of a closed aspherical manifold $M$ with centerless fundamental group so that either

a) there exists a self homotopy equivalence $t: M \rightarrow M$ which generates an element of order $k$ in Out $\pi_{1}(M)$, or

b) there exists a homeomorphism $H: M \rightarrow M$ so that $H_{*}$ generates an element of order $k$ in $\operatorname{Out}\left(\pi_{1}(M)\right)$,

and $t$ (or $H$ ) can not be deformed (or isotoped) to a homeomorphism $K: M \rightarrow M$ with $K^{k}=$ identity.

It is definitely possible to state a positive algebraic result which is the algebraic analogue of the result alluded to earlier in [3].

Theorem 3. Suppose $V$ is a torsion free normal subgroup of a group $K$. Assume that every element of $K$ centralizing $V$ and $K / V$ lies in $V$.

Let $G$ be a finite group and $\varphi: G \rightarrow$ Aut $K$ a function such that

1) $\varphi(G)$ centralizes $V$ and $K / V$;

2) the $\operatorname{map} \psi: G \stackrel{\varphi}{\rightarrow}$ Aut $K \rightarrow$ Out $K$ is a homomorphism.

Then there exists an extension corresponding to the abstract kernel $(G, K, \psi)$.

We have included a (purely algebraic) proof of this result in an appendix. It may be noted from the first lemma that part of the hypothesis may be dropped when $G$ is cyclic. Also the proof of the theorem shows that its conclusion may be strengthened when $G$ is a subgroup of Out $K$ : there exists an extension $E$ with an abelian subgroup whose image is $G$ under $E \rightarrow G$. See statement $\left({ }^{*}\right)$ in the proof for more details.

Appendix. The notation and terminology for the proof of Theorem 3 is taken from [4].

Lemma 1. Suppose $V$ is a torsion-free normal subgroup of a group $K$. Let $G$ be a finite cyclic group and $\varphi: G \rightarrow$ Aut $K$ a function such that

1) $\varphi(G)$ centralizes $V$ and $K / V$;

2) The map $\psi: G \stackrel{\oplus}{\rightarrow}$ Aut $K \rightarrow$ Out $K$ is a group homomorphism.

Then there exists an extension corresponding to the abstract kernel $(G, K, \psi)$.

Proof. Let $k \mapsto \bar{k}$ denote the $\operatorname{map} K \rightarrow \bar{K}=\operatorname{Inn} K \subset$ Aut $K$.

Let $x \in \varphi(G)$ be a generator for $\bar{K} \varphi(G) / \bar{K}$, of order $r$ in the quotient. Thus $x^{r}=\bar{k}$ for some $k \in K$.

Let $x(k)=v k$ where $v \in V$. Then $k=\bar{k}(k)=x^{r}(k)=v^{r} k$. Hence $v^{r}=1$ and so $v=1$ since $V$ is torsion-free. Thus $x$ fixes $k$.

It follows that there is an extension $E$ of $K$ by $\mathbb{Z}_{r}$ such that $E=\langle K, \tilde{x}\rangle, \tilde{x}$ acts by conjugation on $K$ like the automorphism $x$, and $\tilde{x}^{r}=k[4 ;$ th. 15.3.1, p. 225]. 
To get an extension corresponding to $(G, K, \psi)$ just take the pullback of $G$ and $E$ with respect to the common quotient $\mathbb{Z}_{r}$.

Lemma 2. Suppose $V$ is a normal subgroup of a group $K$. Then the group of all automorphisms of $K$ which fix each element of $V$ and $K / V$ is naturally isomorphic to the group of 1-cocycles $Z^{1}(K / V, 8(V))$. In particular, this group is abelian.

Proof. Let $x$. be such an automorphism and put $x(k)=\gamma(k) k$ for $k \in K$. Thus $\gamma(k) \in V$ and $\gamma(v)=1$ for $v \in V$.

From $x\left(k_{1} k_{2}\right)=x\left(k_{1}\right) x\left(k_{2}\right)$ we have $\gamma\left(k_{1} k_{2}\right)=\gamma\left(k_{1}\right) k_{1} \gamma\left(k_{2}\right) k_{1}^{-1}$. In particular $\gamma(k v)=\gamma(k) k \gamma(v) k^{-1}=\gamma(k)$ for $v \in V$ and $k \in K$. Also

$$
\gamma(v k)=\gamma(v) v \gamma(k) v^{-1}=v \gamma(k) v^{-1}
$$

On the other hand $\gamma(v k)=\gamma\left(k k^{-1} v k\right)=\gamma(k)$. Hence $\gamma(k)$ belongs to the center of $V$, and $\gamma(k)$ is independent of the coset representative for $k V=V k$. That is, $\gamma$ is an element of $Z^{1}(K / V, 8(V))$.

Conversely, any such $\gamma$ gives rise to an automorphism of the specified type, and it is clear that the correspondence is an isomorphism of abelian groups.

Proof of Theorem 3. Choose a function $\tilde{\varphi}: \psi(G) \rightarrow \varphi(G) \subseteq$ Aut $K$ such that the composite $\tilde{\psi}: \psi(G) \rightarrow$ Aut $K \rightarrow$ Out $K$ is the inclusion map. Then $\psi(G), \tilde{\varphi}$ satisfy the hypothesis and it is enough to show that the abstract kernel $(\psi(G), K, \tilde{\psi})$ has an extension (see the last line of the proof of Lemma 1).

Hence we may assume at the start that $G \subseteq$ Out $K$ and that $\psi$ is the inclusion map. In this situation we can prove a slightly stronger result (and indeed it is essential to do so):

There exists an extension $E$ corresponding to the abstract kernel $(G, K, \psi)$ such that the associated map $E \rightarrow G$ has a section $x \mapsto \bar{x}$ with $\bar{x} \bar{y}=\bar{y} \bar{x}$ for all $x, y \in G$, and $\bar{x}$ inducing $\varphi(x)$ in the action of $x$ by conjugation on $K \subseteq E$.

We shall prove $(*)$ by induction on the order of $G$. If $G=1$ this is trivial so we may assume $G \neq 1$. By Lemma $2, G$ is abelian; in particular there exists a subgroup $G_{1}$ of prime index in $G$. By induction there exists an extension $E_{1}$ of $K$ satisfying (*) with respect to $G_{1}, \varphi_{1}, \psi_{1}$ where $\varphi_{1}, \psi_{1}$ denote the restrictions of $\varphi, \psi$ to $G_{1}$.

Let (,) be the factor set associated with the section $x \mapsto \bar{x}$. That is,

$$
\bar{x} \bar{y}=(x, y) \overline{x y}, \quad x, y \in G_{1} .
$$

The elements $(x, y)$ belong to the subgroup $K$ of $E_{1}$; moreover they even belong to $V$ by hypothesis, since $(x, y)$ centralizes $V$ and $K / V$, by the equation above and the fact that conjugation by any $\bar{x}$ induces $\varphi(x)$ on $K$.

Next choose an element $s \in G, s \notin G_{1}$; thus $G=\left\langle G_{1}, s\right\rangle$. Define a map $\sigma: E_{1} \rightarrow E_{1}$ by

$$
\sigma(k \bar{x})=\varphi(s)(k) \bar{x} \quad\left(k \in K, x \in G_{1}\right)
$$


Then $\sigma$ is in fact an automorphism of $E_{1}:($ We abbreviate $\varphi(s)=t$.)

$$
\begin{aligned}
\sigma\left(k_{1} \bar{x}_{1}\right) \sigma\left(k_{2} \bar{x}_{2}\right) & =t\left(k_{1}\right) \bar{x}_{1} t\left(k_{2}\right) \bar{x}_{2}= \\
& =t\left(k_{1}\right) \bar{x}_{1} t\left(k_{2}\right) \bar{x}_{1}^{-1}\left(x_{1}, x_{2}\right) \overline{x_{1} x_{2}}= \\
& =t\left(k_{1}\right) t\left(\bar{x}_{1} k_{2} \bar{x}_{1}^{-1}\right)\left(x_{1}, x_{2}\right) \overline{x_{1} x_{2}}=
\end{aligned}
$$

(since conjugation by $\tilde{x}_{1}$ induces $\varphi\left(x_{1}\right)$ on $K$, and $\varphi\left(x_{1}\right)$ commutes with $\varphi(s)=t$ by Lemma 2)

(since $\left(x_{1}, x_{2}\right)$ lies in $V$ )

$$
=t\left(k_{1}\right) t\left(\bar{x}_{1} k_{2} \bar{x}_{1}^{-1}\right) t\left(\left(x_{1}, x_{2}\right)\right) \overline{x_{1} x_{2}}=
$$

$$
\begin{aligned}
& =t\left(k_{1} \bar{x}_{1} k_{2} \bar{x}_{1}^{-1}\left(x_{1}, x_{2}\right)\right) \overline{x_{1} x_{2}}= \\
& =\sigma\left(k_{1} \bar{x}_{1} k_{2} \bar{x}_{1}^{-1}\left(x_{1}, x_{2}\right) \overline{x_{1} x_{2}}\right)= \\
& =\sigma\left(k_{1} \bar{x}_{1} k_{2} \bar{x}_{2}\right) .
\end{aligned}
$$

Futhermore we claim that if $s^{r}$ lies in $G_{1}$ then $\sigma^{r}$ is an inner automorphism of $E_{1}$ : We have $\sigma^{r}(k \bar{x})=\varphi\left(s^{r}\right)(k) \bar{x}$. Since the projections of $\varphi(s)^{r}$ and $\varphi\left(s^{r}\right)$ have the same image $\psi(s)^{r}=\psi\left(s^{r}\right)$ in Out $K$, we have

$$
\varphi(s)^{r} \varphi\left(s^{r}\right)^{-1}=\text { conjugation by } v
$$

for some $v \in K$; in fact $v \in V$ since $\varphi(s)$ and $\varphi\left(s^{r}\right)$ centralize $V$ and $K / V$. Observe that

$$
v \tilde{x} v^{-1}=v \bar{x} v^{-1} \bar{x}^{-1} \bar{x}=v \varphi(x)\left(v^{-1}\right) \bar{x}=v v^{-1} \bar{x}=\bar{x}
$$

also $\overline{s r} \bar{x} \overline{s r}-1=\bar{x}$ by $\left(^{*}\right)$. Hence

$$
\begin{aligned}
(v \overline{s r}) k \bar{x}\left(v \overline{s^{r}}\right)^{-1} & =v \overline{s^{r}} k \overline{s^{r}}-1 v^{-1} \bar{x}= \\
& =v \varphi\left(s^{r}\right)(k) v^{-1} \bar{x}= \\
& =\varphi(s)^{r}(k) \bar{x}= \\
& =\sigma^{r}(k \bar{x}),
\end{aligned}
$$

and so $\sigma^{r}$ is an inner automorphism of $E_{1}$ as claimed.

Define a function $\varphi_{2}: G / G_{1} \rightarrow$ Aut $E_{1}$ by sending the image of $s^{i}$ in $G / G_{1}$ to $\sigma^{i}$, $0 \leqq i<\left|G / G_{1}\right|$, and let $\psi_{2}$ be the composite $G / G_{1} \rightarrow$ Aut $E_{1} \rightarrow$ Out $E_{1}$. The map $\psi_{2}$ is a homomorphism of groups by the preceding paragraph. The hypotheses of Lemma 1 are clearly satisfied, and so there exists an extension $E_{2}$ of $E_{1}$ corresponding to the abstract kernel $\left(G / G_{1}, E_{1}, \psi_{2}\right)$.

Now let $\eta$ be the map $E_{2} \rightarrow$ Aut $E_{1}$ induced by conjugation. By construction $\eta\left(E_{2}\right)=\left\langle\eta\left(E_{1}\right), \sigma\right\rangle$. Next consider the map $\mu:\left\langle\eta\left(E_{1}\right), \sigma\right\rangle \rightarrow$ Aut $K$ induced by restriction to $K$. By construction of $E_{1}$ we have $\mu\left(\eta\left(E_{1}\right)\right)=\varphi\left(G_{1}\right) \operatorname{Inn} K$; also we have by definition of $\sigma$ that $\mu(\sigma)=\varphi(s)$.

Since $G=\left\langle G_{1}, s\right\rangle$ we have an epimorphism $E_{2} \rightarrow \varphi(G)$ Inn $K \rightarrow G$ with kernel containing $K$; in fact the kernel must be equal to $K$ since

$$
\left|E_{2} / K\right|=\left|E_{2} / E_{1}\right|\left|E_{1} / K\right|=\left|G / G_{1}\right|\left|G_{1}\right|=|G| \text {. }
$$

Obviously the diagram

$$
\begin{aligned}
& G \stackrel{\psi}{G} \text { Out } K \\
& \uparrow \\
& E_{2} \rightarrow \text { Aut } K
\end{aligned}
$$


is commutative, since the composite $\mu \eta: E_{2} \rightarrow \varphi(G) \operatorname{Inn} K$ sends each element of $E_{2}$ to the element of Aut $K$ it induces by conjugation on $K$. Thus $E=E_{2}$ is an extension of $K$ corresponding to the abstract kernel $(G, K, \psi)$.

It remains to produce a section for $E \rightarrow G$ satisfying (*). Let $\bar{s} \in E_{2}$ be chosen so that $\bar{s}$ induces $\sigma$ by conjugation on $E_{1}$; it follows that $\bar{s} \bar{x} \bar{s}^{-1}=\sigma(\bar{x})=\bar{x}$ for $x \in G_{1}$. Obviously the group $S=\left\langle\bar{s}, \bar{x} \mid x \in G_{1}\right\rangle$ is commutative and induces $\langle\varphi(G)\rangle$ by conjugation on $K$. Now just pick $\bar{x}$, for $x \in G$, to be an element of $S$ which induces $\varphi(x)$; these choices may even be made consistent with previous notation though that is not required. The map $x \rightarrow \bar{x}, x \in G$, is automatically a section for $E \rightarrow G$ since the image of $x$ under $E_{2} \rightarrow \varphi(G) \operatorname{Inn} K$ is $\varphi(x)$ and the image of $\varphi(x)$ under $\varphi(G) \operatorname{Inn} K \rightarrow G$ is $x$. Clearly this section satisfies (*). Q.E.D.

\section{References}

[1] P. E. Conner and F. Raymoxd, Manifolds with few periodic Homeomorphisms. Proc. of the second Conference on Compact Transformation Groups, LNM 299, Berlin-Heidelberg-New York 1970.

[2] P. E. Conner and F. RAymond, Realizing finite groups of homeomorphisms from homotopy classes of self-homotopy-equivalences. Manifolds-Tokyo 1973, edited by Akio Hattori, Tokyo $1975,231-238$.

[3] P. E. Conver and F. Ruymond, Deforming homotopy equivalences to homeomorphisms in aspherical manifolds. Bull. Amer. Math. Soc. 83, 36-85 (1977).

[4] M. HaLl, The Theory of Groups. New York 1959.

[5] O. HANNER, Some theorems on absolute neighborhood retracts. Ark. Mat. 1, 389-408 (1951).

[6] J. Nizisen, Abbildungsklassen endlicher Ordnung. Acta Math. 75, 23-115 (1913).

[7] J. L. Tollefson, Periodic homeomorphisms of 3-manifolds fibered over $S^{1}$. To appear.

[8] H. Zirschang, On extensions of fundamental groups of surfaces and related groups. Bull. Amer. Math. Soc. 80, 366-367 (1974).

Eingegangen am 20.9.1975*)

Anschrift der Autoren:

Frank Raymond

Department of Mathematics

University of Michigan

Ann Arbor, Michigan 48109, USA
Leonard L. Scott

Department of Mathematics

University of Virginia

Charlottesville, Virginia, USA

*) Eine revidierte Fassung ging am 20.1.1977 ein. 